\title{
L'espace scénique comme représentation de l'espace dramatique dans Le Chien de Jean Marc Dalpé
}

\section{Mariel O’Neill-Karch}

(Université de Toronto)

"Pour moi, le travail le plus difficile demeurait la mise en scène. Il fallait trouver les modes de transition adéquats pour assurer une cohésion à ce condensẻ de la vie d'une famille où s'entremêlent passé et présent." Jean Marc Dalpé,

L'Express de Toronto (19-25 avril 1988).

"Le texte de theâtre a besoin pour exister d'un lieu, d'une spatialité où se déploient les rapports physiques entre les personnages."

Anne Ubersfeld. Lire le théâtre. p. 166.

Dans un passage bien connu de Notes et contre-notes Ionesco affirme que "tout est langage au théâtre, les mots, les gestes, les objets, l'action elle-même, car tout sert à signifier., ${ }^{1}$ Dans un texte encore plus souvent cité, Roland Barthes avait déjà souligné la simultanéité des messages transmis par ces divers langages: "en tel point du spectacle, vous recevez en même temps six ou sept informations (venues du décor, du costume, de l'éclairage, de la place des acteurs, de leurs gestes, de leur mimique, de leur parole)" qu'il résume dans une formule lapidaire, "une épaisseur de signes.»"

Le thêâtre est donc un ensemble de signes/langages qui, conjugués ensemble, émettent un message complexe transmis par des moyens auditifs (parole, musique, bruitage) et visuels (décor, costumes, accessoires, éclairage, etc.), ce message ayant un contenu intellectuel qu'il est possible de verbaliser, du moins en grande partie. Le théâtre transmet surtout, cependant, des messages à 
contenu artistique, des messages qui disent que ce que l'on voit, que l'on entend, c'est du théâtre, et qui procurent au spectateur, grâce à l'organisation des divers signes, un plaisir esthétique.

Ce plaisir vient souvent du travail du metteur en scène et du scénographe qui unissent leurs efforts pour dialoguer avec le texte ou pour créer un nouveau texte dont un des langages particulièrement significatifs est le décor qui s'apparente étroitement à des éléments qui distinguent le théâtre d'autres formes d'expression artistique comme l'espace scénique et la présence corporelle de comédiens. Dans notre étude du Chien ${ }^{3}$ de Jean Marc Dalpé, nous verrons comment les nombreux signes textuels de l'espace, ou espace dramatique, se traduisent par d'autres signes, ceux de l'espace réel de la scène, ou espace scénique (dont fait partie le décor) et ceux de l'espace créé par les comédiens et le metteur en scène, ou espace ludique.

Le Théâtre du Nouvel-Ontario, en collaboration avec le Centre d'essai des auteurs dramatiques, a présenté Le Chien en lecture publique le 3 septembre 1987, à Québec, dans le cadre du Sommet de la francophonie. C'est donc sans décor que le premier public a reçu cette pièce dont l'intrigue se résume comme suit: le retour d'un fils après "sept ans de trips de fou d'un bout à l'autre du Canada pis des États" et un règlement de comptes avec sa brute de père, ce qui n'a rien d'original, mais c'est rarement au niveau de l'intrigue qu'il faut chercher l'originalité.

C'est plutôt par le biais de la structure que Jean Marc Dalpé a réussi à rendre le drame intéressant, car il a su planter ça et là des bombes à retardement qui font éclater la chronologie et brouiller les pistes de telle sorte que nous assistons non seulement au retour de l'enfant prodigue, mais aussi à des scènes du passé où l'on voit la mère désabusée, le grand-père qui vient de mourir et la soeur adoptive dont le récit déclenchera le geste final de Jay.

Dans un texte qui remplit la fonction de mise en abyme, la mère utilise une comparaison avec une série de photos qui se présentent de façon achronologique:

Mais dans ma tête... dans ma p'tite tête ...j' nous vois le jour du mariage pis, drette â côté ... drette à côté, j'me vois où c'est que j'suis rendue 
aujourd'hui. Ca s'allume pis ça s'éteint d'un bord pis de l'autre. Tout autour de ça, y'a d'autres photos qui viennent, qui partent ... de quand j'étais jeune fille, de quand t'es né, de quand y t'frappait ... des fois ça va vite, vite, vite, pis j'suis toute mêlée en dedans (19).

Ceci fait écho à la structure en mosaïque de la pièce où plusieurs scènes fortes du passé trouvent leur place dans ce texte éclaté, fragmenté. Ce n'est donc pas tant d'intrigue qu'il faudrait parler, que de tension soutenue par un habile tissage de fils.

Cette tension se concentre, l'espace de temps que dure la confrontation père-fils, à l'orée d'un bois, dans le nord de l'Ontario, devant lá demeure du père de Jay. Voici comment Dalpé décrit ce décor: "La cour en avant d'une maison mobile au bout d'un petit chemin de terre, près du village" (6), ce qui attire l'attention du lecteur sur quatre aspects significatifs de l'espace: la cour, la maison mobile, le bout d'un petit chemin de terre et la proximité du village.

Que ce soit dans la cour et non dans la maison qu'a lieu la rencontre entre les deux hommes révèle plusieurs choses. La plus évidente, c'est qu'il fait chaud, l'action se passant un soir de juillet. La plus significative, c'est que le père défend son territoire contre son fils qu'il n'est pas du tout heureux de revoir et qu'il n'a pas l'intention de faire entrer dans la maison. L'emplacement de la maison "au bout d'un petit chemin de terre" indique une volonté de vivre isolé de ses semblables, personne ne passant devant la porte puisque la route ne continue pas plus loin. La condition du chemin qui est "petit" et "de terre" est celle de routes primitives, ce qui concorde tout à fait avec le caractère farouche du père qui tient à son isolement qui n'est cependant pas complet puisque la maison se trouve "près du village," ce qui permet au père de se rendre sans difficulté à la taverne toutes les semaines. La maison du père est "mobile," ce qui rappelle les roulottes des romanichels et suggère qu'il n'a pas réussi à s'enraciner dans ce coin du pays fondé par de braves défricheurs, dont son père.

Le grand-père de Jay est donc arrivé dans le nord comme pionnier, rêvant d'un avenir meilleur, celui du propriétaire terrien: 
Une terre. Toé, tu peux pas savoir c' que ça voulait dira ça dans mon Temps. Oh! Tu peux ben penser l'savoir mais tu l'sais pas au fond, pantoute. R'garder d'un bord à l'autre pis pouvoir se dire: "C't'à moé, ça!" quand t'avais été élevé sur des patates pis de la mélasse ..." (32).

Quand le grand-père est devenu trop vieux pour l'exploiter, c'est au fils aîné qu'est passée la terre: "La terre revenait à ton oncle, mon frère. Quand le vieux y’a donnée, moé j'êtais dêjà dans l'bois, pis j'allais pas travailler pour quelqu'un d'autre dessus" (34). Blessé dans son orgueil d'avoir été écarté de la terre paternelle, le père de Jay a refusé plus tard de remplacer son frère, mort prématurément. La terre a donc étè vendue, lopin par lopin, un des terrains servant maintenant de cimetière. On peut alors mesurer toute l'ironie du "C't'à moé, ça!", puisque le jour de la confrontation finale entre Jay et son père est le jour oủ le grand-père a été enterré dans cette terre qui ne lui appartenait déjà plus.

Pourtant le père de Jay, qui a refusé cette terre qu'on lui avait offerte, mais trop tard, n'est jamais arrivé à s'en éloigner et est rempli d'admiration pour son père:

Ça devait être quequ'chose pareil ... arriver dans c'temps-là au milieu du bois. ... A chaque jour, tu vois c' que t'as fait, comment t'as avancé, pis tu t'poses pas de questions, parce que tout est là, drette là, en avant de tes yeux. T'es en train de faire r'culer une forêt, tabarnac! (33).

C'est sans doute à cause de cette admiration qu'il vouait à son père que même après son refus, il a rêvé à la possibilité de racheter la terre et de l'exploiter avec Jay:

PERE: J'aurais voulu qu'on reprenne la terre, la terre de ton grand-père, toé pis moé. La r'partir, la r'bâtir, revoir des affaires pousser dessus. Avec mes bras. Avec tes bras. 
JAY: Ten as jamais parlé avant.

PERE: J'attendais.

JAY: Quoi?

PERE: De toute façon, ça aurait pas pu marcher. Prend trop d'argent astheure. Pis encore plus icitte à cause d'la neige qui peut te tomber sur la tête au mois de juin, pis les gels qui peuvent arriver début septembre. J'étais fou d'y avoir pensé (31).

Il y a pensé, mais sans pouvoir passer à l'action, attendant passivement un signe de Jay, sachant bien, dans son fort intérieur, qu'il n'en viendrait jamais. Il a donc refusé par orgueil, puis par lâcheté, l'héritage de son père qu'il admirait. Et comme Normand Renaud l'a si bien observé, ken renonçant à la terre, il a renoncé à ses plus nobles aspirations. Il s'est dégradé, il a perdu toute estime pour lui-même, pour enfin sombrer dans l'incommunicabilité et la violence autodénigrante. ${ }^{4} \mathrm{Par}$ opposition, la noblesse du grand-père est soulignée par le curé qui rappelle, dans son eulogie, que la photo du défunt se trouve avec celle des autres fondateurs du village sur les murs du bureau de poste. S'il devait y voir une photo du père, elle serait plutôt sur les murs de la taverne, espace déprimant qu'il hante depuis vingt ans.

La mère de Jay a sombré elle aussi dans une profonde dépression centrée sur l'espace qui l'entoure:

J'hais toute icitte. Toute. Nomme-le pis j'l'hais. $J$ 'hais les arbres. Les osties d'épinettes. Rachitiques, grises pis tassées comme dans une canne de sardines. On dirait qu'y s'égorgent, qu'y s'boivent, qu'y s'mangent les unes, les autres ... Pareil comme le monde (34).

Les arbres, symboles de vie, deviennent pour elle des monstres qui peuplent ses cauchemars. La mère est donc réduite à détester cette 
terre qu'elle a "dans les os comme un cancer" (34), à la détester malgré sa beauté:

J'hais les ciels au coucher du soleil à l'automne, pis les aurores boréales, pis les matins de printemps, le jour que tout s'met à dégeler ... parce qu'y sont beaux ces sacraments-là, pis qu'y devraient pas avoir le droit d'être aussi beaux (34).

Jay, dont le nom fait de lui l'incarnation des multiples "j'hais" de sa mère qui l'a mis au monde avec tout son bagage personnel de haine, a senti le besoin de partir pour tenter de rompre les chaînes qui, comme celles du chien, le retenaient attaché à un espace précis. Plus lucide que sa mère, il lui répond qu'«y'a des trous pareils partout, sinon pires même" (34).

Le trou est l'image spatiale exploitée le plus souvent dans le texte, et c'est presque toujours dans un contexte négatif, les images étant surtout grossières ou violentes. Dans la quatrième réplique de la pièce, Jay donne le ton: "Câlice d'ostie d'place de cul!" (7), mêlant blasphèmes à une figuration de l'espace où le trou du cul joue un rôle de premier plan. La mère va enchaîner avec une référence scatologique: "Pis même si y'est né dans un trou de marde comme icitte" (9) à laquelle feront écho Jay (15), le père (26) et le grand-père (28), Dalpé n'épargnant rien pour que le public sente l'importance de l'image, nous la servant même en anglais: "j'y dis le nom du village icitte. 'Where is that for fuck's sake?'... "Ontario, you asshole!" (47), transposant ainsi l'image de l'espace au personnage, mais liant fortement les deux par juxtaposition.

La violence de l'image est associée aux trous faits par des bombes dans les tranchées où le grand-père a passé les années 1914-1918 (28-9) et au trou fait par la balle qu'il a tirée dans la tête d'un jeune Allemand qu'il a surpris (30). Le long récit du grand-père prépare l'introduction d'un nouvel élément, un vieux Luger allemand, souvenir de cette guerre lointaine, arme à feu qui passera du père au fils plusieurs fois au cours de la soirée et qui servira à tuer le chien d'abord, puis le père, deux êtres qui avaient déjà cessé de vivre bien avant l'intervention de Jay qui dit du chien: "Y'est déjà mort! 
Tout c'qui reste là, c'est une boule de nerfs qui souffre" (49), et s'adressant à son père: "Ça fait combien longtemps que t'es mort? Faudrait peut-être que j'le dise à quelqu'un..." (53). Cette mort vivante est assimilée par la mère à l'image du trou: "C'était dans ses yeux. Y'a toujours eu quequ'chose de dur dans ses yeux. Même aux noces. Mais avant, c'était pas gros. Juste comme un petit trou noir où c'est qu'y'avait rien de vivant, pis ou c'est que rien pourrait jamais vivre" (24).

Les morts dont se sentent entourés les personnages doivent être enterrés. C'est le cas du grand-père dont les restes sont "dans le champ qu'y'avait défriché" (13), maintenant transformé en "trou de bouette" (48). Vers la fin de la pièce, quand Jay a compris qu'il n'y avait pas de réconciliation possible avec son père, il parle de partir:

Si je reviens, ça va être pour t'enterrer ... ouain ... m'a revenir t'enterrer. Six pieds en-dessous. Non, même pas. Dix pieds. M'a le creuser le trou moi-même. Pis si j'arrive trop tard, pis qu'y l'ont déjà fait... Sacrament! M’a te déterrer, pis j’vas en creuser un autre. Vingt pieds chriss. Non, plus gros encore. Parce que m'a tout mettre dedans: ton linge, tes outils, toutes tes patentes, tabarnac! M'a enterré ton char pis c'te chriss de maisons-là avec ... (53).

C'est l'ultime trou, celui que contiendrait non seulement le corps du défunt, mais tous les objets qui formaient son monde, même la maison, cette maison mobile que Jay a retrouvée après sept années d'absence:

Trois jours pis trois nuits, pis sept ans, pis y'a rien de changé chriss! Ni toé, ni moé, ni la maison. C'est toujours pareil, pareil. Comme figé dans le roc. Toé d'un bord, moé de l'autre. Comme si j'étais dans un trou de bouette, le même trou de bouette que celui de grand-papa ... (48) 
C'est à partir d'indications comme celles-ci que Pierre Perrault a choisi un principe organisateur, celui du trou, et qu'il a réussi à créer une sorte de condensé rhétorique du texte, un décor hautement significatif par sa géométrie interne et que j'ai pu voir, à Toronto, au Factory Theatre, lors de la production en langue anglaise du Chien. ${ }^{5}$

La scène elle-même était en forme d'amphithéâtre taillé dans le roc du bouclier pré-cambrien. A gauche, quelques marches qui menaient à la porte de la maison mobile du père. Au centre, à l'avant-scène, il y avait un demi-cercle dans lequel se trouvait de la terre mêlée à des cailloux, à l'image même du sol ingrat du nord de l'Ontario. Ces références sans équivoque forment un ensemble signifiant, lié d'abord à la difficulté de vivre là où la nature est si hostile, et aux personnages qui ont été façonnés par ce "trou" et qui lui ressemblent, comme le déplore la mère au sujet des épinettes: "On dirait qu'y s'égorgent, qu'y s'boivent, qu'y s'mangent les unes, les autres ... Pareil comme le monde" (34). Nous ne voyons pas les plantes, mais ce que le spectateur perçoit des êtres humains placés devant lui sur scène confirme les dires de la mère: nourris du même sol, la flore et la faune se comportent de la même façon agressive, axée sur le principe de survivance.

C'est la mère encore qui décrit le "coucher de soleil à l'automne, pis les aurores boréales pis les matins de printemps" (34) qu'elle hait parce qu'ils sont trop beaux. C'est cette beauté que les artisanscréateurs du Chien ont essayé de transmettre par un éclairage varié, projeté sur une toile de fond contre laquelle se découpent les personnages qui semblent taillés, eux aussi, dans le roc.

Malgré des éléments très concrets: la porte de la maison mobile, les quelques marches qui y mènent, la roche, la terre, et un éclairage qui reproduit le ciel sur une toile de fond, la partie surélevée du décor reste singulièrement vide, ce qui cadre tout à fait avec le travail de scénographie moderne fait ailleurs. Jay et son père se situent dans l'espace concret, et c'est dans cet espace que paraissent certains accessoires: le sac de voyage de Jay, la caisse de douze bières qu'il apporte avec lui, le sac du père dans lequel se trouve le vieux Luger allemand. L'espace où se meuvent les autres personnages: le grandpère mort, la mère, la soeur adoptive Céline, est un espace psychique qui figure les souvenirs, les obsessions de Jay qui prennent le dessus quand la conversation avec son père ne va nulle part. 
Il est donc évident que le scénographe Pierre Perrault a travaillé à partir du texte de Dalpé d’où il a puisé les images qui lui ont servi dans la conception du dispositif scénique conçu pour des théâtres avec scène dans le style italien, c'est-à-dire avec le public d'un côté seulement. Ce décor très pensé à été compris par le public, si on en juge d'après certains des critiques qui en ont parlé. Normand Renaud a vu, par exemple, que cette aire "en forme de cuvette sablonneuse que jamais le père ne réussira à quitter, rapelle avec pertinence le trou d'obus du récit de guerre du grand-père. ${ }^{6}$ Philip Wickham, pour sa part, a interprété le décor "comme un véritable entonnoir de passions en feu et chaque personnage, sous un ciel flamboyant, se tient au bord du gouffre dans lequel le père est tombè." ${ }^{7}$ C'est donc la forme même du décor, cette sorte de fosse aux lions où a lieu un combat à mort, qui a retenu l'attention.

Il y a cependant d'autres éléments qui sont aussi d'une grande importance, comme la matérialité mème de ce décor, que nous avons déjà analysée, le corps des comédiens qui font partie intégrante de l'espace et dont nous allons parler en traitant de mise en scène, l'éclairage et enfin, la musique. C'est ce qu'a constaté Jacques Morlaud, en rendant compte de la production du Chien au Festival des Francophonies à Limoges: "Au jeu des artistes, au texte, à la musique, il ne faut pas dissocier l'éclairage soigné et le décor adopté imaginé comme sur un plateau de tournage ciné." ${ }^{8}$

L'espace visuel créé par le décor est rendu plus fort encore grâce à un dispositif sonore, conçu et interprété par Robert Paquette, qui consiste en trois parties distinctes qui s'unissent pour former un tout.

Premièrement, aux temps forts, il y a le chien qui grogne, jappe, aboie, clabaude, clatit, glapit, hurle et gronde. Ce chien que l'on ne voit jamais fait sentir sa présence comme celle d'une force primitive enchaînée qui ne demande qu'à être libérée. L'animal, qui occupe d'abord un espace sonore, occupe aussi un espace intérieur, celui du fantasme, du rêve, du cauchemar, comme l'ont compris certains critiques. Pour Léo Beaulieu, "il est tout ce qui fait peur, qui nous fige et nous empêche d'agir, il est un passé avilissant et lourd à supporter, une condition impossible à supporter, une condition impossible à fuir en voyageant ou par l'oubli." ${ }^{9}$ Pierre Roberge a vu, dans le chien, "l'être humain enchaîné à sa condition, qui en devient 
fou et qu'il faut abattre." ${ }^{10}$ D'ailleurs, dans certaines traditions, l'aboiement d'un chien près d'une maison est présage de mort. On nous apprend qu'il est attaché, qu'il occupe donc un espace fort restreint, ce qui l'apparente aux personnages qui, chacun à sa façon, souffrent d'une entrave à leur liberté personnelle.

Deuxièmement, il y a de temps en temps un son continu, comme un mantra, qui suggère la méditation ou la participation à un rite initiatique. Cela sert à nous séparer du monde de tous les jours et à nous faire pénétrer dans un espace très précis, très spécial, celui du monde intérieur de Jay.

Troisièmement, il y a Robert Paquette qui joue sa guitare en contrepoint avec le dialogue, le ponctue, l'amplifie, le remplace même parfois. Il évoque l'atmosphère, suggère une tonalitè, s'ètire pour lier un personnage à un autre, dialogue avec le texte. Le fait qu'il s'agisse d'une guitare électrique nous plonge en plein continent nordaméricain, cette vaste étendue que Jay a parcourue pendant sept ans avant de revenir, faire face à son père.

La mise en scène de Brigitte Haentjens était dépouillée, sobre, et significative, utilisant au maximum le décor de Pierre Perrault et incorporant la musique de Robert Paquette pour créer un espace ludique, le théâtre étant par définition là oủ jouent des comédiens. L'espace théâtral est donc à la fois statique et dynamique, inanimé. C'est dans les rapports entre ces axes que se situe, en partie du moins, le travail de mise en scène.

Brigitte Haentjens a utilisé les interprètes comme des composantes d'un espace ludique d'abord, car les personnages paraissent dans un décor vide qui les théâtralise, et que fait accepter que même si les personnages ne se trouvent pas litteralement au même endroit, les interprètes sont devant nous et ils réagissent les uns aux autres, se parlent, se touchent, se sautent même dessus. Dans une scène particulièrement réussie, Céline, la soeur adoptive de Jay, s'accroche au cou de son frère et lui grimpe littéralement sur le dos, position qu'elle maintient pour lui raconter assez longuement le plaisir qu'elle avait éprouvé à relire les cartes postales qu'il avait envoyées à leur mère au cours de ses sept années d'absence. Brigitte Haentjens utilise donc les interprètes comme des éléments d'une sculpture mobile, des participants à un jeu visuel, les faisant bouger de telle sorte qu'ils créent des images scéniques d'une très grande force. 
Dans cette dynamique, le père et le fils se tiennent presque toujours au premier plan, comme l'a bien observé Normand Renaud:

La tension dans les rapports entre les personnages se traduit par le jeu des positions qu'ils occupent les uns par rapport aux autres sur le plancher incliné de la scène. Alors que les évocations d'incidents passés viennent des personnages situés vers le haut et la droite de la scène, les affrontements du père et du fils dans le présent réel de la pièce ont tous lieu au premier plan à gauche, au point le plus bas de la scène. L'inclination (sic) de la scène a donc fourni un moyen discret mais habile de suggérer visuellement les décalages chronologiques de laction. $^{11}$

Non seulement les décalages chronologiques, mais aussi les rapports entre personnages sont donc suggérés par la position des interprètes sur la scène inclinée. Ce que regarde le spectateur est hiérarchisé de telle sorte qu'il comprend tout de suite les divers champs de force que Brigitte Haentjens a voulu mettre en valeur. Il est fort intéressant de noter, par exemple, que Jay et son père, qui se tiennent vers le bas de la scène, tournant autour du petit cercle de terre et de cailloux, s'asseyant pour boire cannette sur cannette de bière, sont plutôt dans le domaine de l'horizontalité, leur gestuelle étant liée à la terre que Jay manie et aux cailloux qu'il lance par dépit. Les personnages qui peuplent le psychisme de Jay se dressent debout, loin de la terre, appartenant à la verticalité et suggérant la possibilité d'élévation, d'évasion.

Anne Ubersfeld a dẻjà analysé pareils liens entre espace et psychisme, notant que "l'espace scénique peut aussi apparaître comme un vaste champ psychique où s'affrontent des forces qui sont les forces psychiques du moi. La scène est alors assimilable à un champ clos où s'affrontent les éléments du moi divisé, clivé." ${ }^{12}$ Il est bien sûr vrai que chaque personnage est le centre d'un monde psychique, mais il y a de nombreux indices dans Le Chien qui montrent que c'est le monde de Jay qui est privilégié. Il est le premier 
à paraître sur scène, et sa rencontre avec son père, sa bête noire, après sept années d'absence est au coeur du drame, et se fait au centre du plateau, les deux hommes se trouvant au même niveau, comme deux gladiateurs dans un amphithéâtre. Les autres personnages, comme nous, sont les spectateurs d'un drame qui est en partie le leur, mais leur position dans le vide les empêche d'y participer directement. La mise en scène permet donc au spectateur de saisir ce rapport de forces qui restera stable durant toute la représentation, tout en s'intensifiant jusqu'au geste final.

Le geste ultime de Jay qui se produit sur scène, devant les spectateurs, est le dernier d'une série de gestes violents qui ponctuent Le Chien. Il y a d'abord les gestes du passé que l'on ne voit pas mais dont on parle. Nous avons déjà mentionné le récit que fait le grand-père de la mort qu'il infligea à un jeune soldat allemand, violence cautionnée par la société qui a inventé la guerre pour assouvir le goût du sang. Le père, qui n’a pas cet échappatoire, s'attaque à ceux et celles qui l'entourent: "C'est comme un feu qui s'allume icitte. Ça me brûle dans poitrine, pis y faut que j'fesse sur quequ'chose ou sur quelqu'un pour que ça l'arrête ... pour que ça s'éteigne" (50), explique-t-il, mais sans s'attirer la sympathie de sa famille qui doit toujours encaisser. N'y tenant plus, Jay s'est un jour tourné contre son père à qui il a "fourré une volée" (16) et juste avant de quitter la maison, a lancé cette phrase prophétique: "M'a l'tuer si j'le revois" (16).

Pendant les sept années qu'a duré l'absence de Jay, d'autres incidents se sont produits. Après avoir frappé sa femme assez fort pour qu'elle soit obligée de s'acheter un dentier, c'est au "chum" de celle-ci qu'il s'en prend: "Ton père l'a réveillé au milieu de la nuit, y'a mis sa 22 entre les deux yeux, y'a pesé sur la gâchette pis y'a fait "Bang!" comme ça avec sa bouche" (18). Le lendemain matin, l'homme était parti. Céline aussi se fait battre, mais les visées du père sont tout autres puisque cela se termine par un viol (61). Ce sont donc les gestes du passé qui viennent informer le présent, le vieux Luger du grand-père servant à Jay pour venger la violence faite contre sa mère et sa soeur. A la fin, l'espace de la vie et l'espace de la mort se rejoignent quand "le grand-père reprend le fusil des mains de Jay" (62). 
Le Chien témoigne de la maturation du théâtre franco-ontarien qui, avec cette pièce, s'insère dans la tradition réalistico-poétiqueen-blue-jeans du cinéma et du théâtre de John Steinbeck et de Sam Shepard, espace américain que Jay transporte désormais avec lui et qui entre en collision avec l'espace de son passé, avec l'espace de son père. Mais l'espace américain est difficile à définir:

MERE: C'était beau la Californie?

JAY: Pas disable.

MERE: Essaie.

JAY: Comme dans les vues.

MERE: Comme sur les cartes postales?

JAY: Pareil (25).

Ayant admiré tout ce qu'il a vu, mais n'ayant pu pénétrer dans l'espace des cartes postales, celui d'une autre culture, Jay est revenu chez lui, à la frontière d'un pays à faire/refaire, qui ne lui appartient pas/plus et face auquel il doit pourtant se définir. C'est la réalité des Franco-Ontariens et des Québécois qui se sont tout de suite reconnus dans cette pièce.

Le Chien, c'est encore du théâtre très littéraire, dans ce sens qu'il y a, dans le texte publié de Jean Marc Dalpé, très peu de didascalies. Mais l'apport certain des dispositifs scéniques de Pierre Perrault et sonores de Robert Paquette et la subtilité de la mise en scène de Brigitte Haentjens en ont fait un spectacle où divers langages se conjuguent pour arriver à "une épaisseur de signes." 


\section{Notes}

${ }^{1}$ Eugène Ionesco, Notes et contre-notes (Paris: Gallimard, 1966), p. 116.

${ }^{2}$ Roland Barthes, Essais critiques (Paris: Seuil, 1964), p. 258.

${ }^{3}$ Le Chien de Jean Marc Dalpé, une co-production du Théâtre du Nouvel-Ontario et du Théâtre français du Centre national des Arts, a été créé à Sudbury le 24 fêvrier 1988, à la Caverne de Science Nord, dans une mise en scène de Brigitte Haentjens. Décor et costumes: Pierre Perrault. Musique: Robert Paquette. Distribution: Roger Blay (le père), Roy Dupuis (Jay), Hélène Paulin (Céline), Marthe Turgeon (la mère) et Lionel Villeneuve (le grand-père). Le Chien a été publié par les éditions Prise de Parole. Les chiffres entre parenthèses dans le texte renvoient à la première édition, celle de 1987.

${ }^{4}$ Normand Renaud, "Le Chien de Dalpé: Une grande oeuvre. Il faut tuer le chien," L'Original déchaîné, vol. 1, no 10 (1er mars 1988), p. 11.

${ }^{5}$ Le Chien traduit en anglais par Maureen Labonté, a tenu l'affiche au thêâtre Factory du 11 novembre au 4 décembre. Isabelle Vincent remplaçait Hélène Paulin dans le rôle de Céline.

${ }^{6}$ Normand Renaud, "Le Chien monté par le T.N.O.: Une production remarquable," L'Original déchaîne, vol. 1, no 10 (1er mars 1988), p. 10.

${ }^{7}$ Philip Wickham, "Le Chien: Le meilleur ennemi de l'homme," Continuum (semaine du 14 mars 1988), p. 23.

${ }^{8}$ Jacques Morlaud, L'Écho du texte, cité dans L'Express de Toronto (semaine du 24 au 30 octobre 1989), p. 5.

${ }^{9}$ Léo Beaulieu, "Le Chien: Un Pit Bull franco-ontarien," L'Express de Toronto (semaine du 15 au 21 novembre 1988), p. 9. 
${ }^{10}$ Pierre Roberge, «Le Chien: La primauté absolue du texte sur la scène," La Presse (5 mars 1988).

${ }^{11}$ Normand Renaud, "Le Chien monté par le T.N.O.: Une production remarquable," L'Original déchaîné (1er mars 1988), p. 10.

${ }^{12}$ Anne Ubersfeld, Lire le théâtre (Paris: Éditions Sociales, 1977), p. 170 . 